\title{
A Millimeter-Scale Electrostatic Flapping-wing Actuator with High Lift-to-Weight Ratio
}

\author{
Yi Yang ${ }^{1, a *}$ \\ ${ }^{1}$ School of Energy and Power Engineering, Beihang University, Beijing 100191, China \\ ayangyi@buaa.edu.cn
}

Keywords: FMAV actuator, Electrostatic, Millimeter-scale, Lift-to-weight ratio

\begin{abstract}
We present a millimeter-scale, 3mg-weight electrostatic flapping-wing actuator which can generate lift force of $1.5 \mathrm{mg}$ under a stroke frequency of $80 \mathrm{~Hz}$, and makes the lift-to-weight ratio reach 0.5. As such, this work makes a key step in achieving electrostatic actuated FMAV's autonomous flight.
\end{abstract}

\section{Introduction}

Insect-inspired flapping-wing micro air vehicles (FMAVs) are centimeter-scale, or even millimeter-scale artificial flying insects. One of the greatest challenges in FMAV research is the design of an actuator that is both suitable and efficient in a small scale. Several kinds of actuators, such as electrostatic actuators [1], electromagnetic actuators [2] or piezoelectric actuators [3], have been researched preliminarily. With its advantages in power consumption and miniaturization, the electrostatic actuator has great potential as a millimeter-scale actuator for FMAVs. However, previous electrostatic actuator, such as the one demonstrated in [1], can only generate $3.1 \mathrm{mg}$ lift force. As the total weight is $330 \mathrm{mg}$, the lift-to-weight ratio of that prototype is 0.0094 , which means autonomous flight is still unable to achieve at present.

In this paper, we propose a single-wing configuration which works in self-resonance state under the electrostatic field. Moreover, a fabrication methodology using laser to cut carbon fiber, polymer membrane, and tinfoil, is also discussed. Based on the above content, a millimeter-scale flapping-wing actuator with similar size as mosquitoes weighs only $2 \mathrm{mg}$ is presented. As shown in forthcoming sections, the lift-to-weight ratio of this actuator is 0.5 , which is a key step in the realization of the electrostatic-actuated FMAVs' total autonomous flight.

\section{Design}

Fig. 1(a) shows the conceptual drawing of the actuator. The actuator consists of five primary components: the base, metal beam, a pair of electrodes, wing hinge, and wing. The function of each component can be described as follows: 1) the base provides an insulated substrate to the metal beam and joins the two electrodes with each other; 2) the metal beam, on which through-hole arrays spread for the purpose of decreasing aerodynamic drag and increasing the vibration frequency, vibrates in working state and outputs displacement at the beam tip; 3) the electrodes are connected respectively to the positive and negative pole of a DC power source, and should provide a steady electrostatic field throughout the space between them; 4) the wing hinge is flexible and allows the wing to passively rotate while working; and 5) the wing must stroke at an stroke angle and rotation angle, so that a lift force may generate on the wing. 
(a)

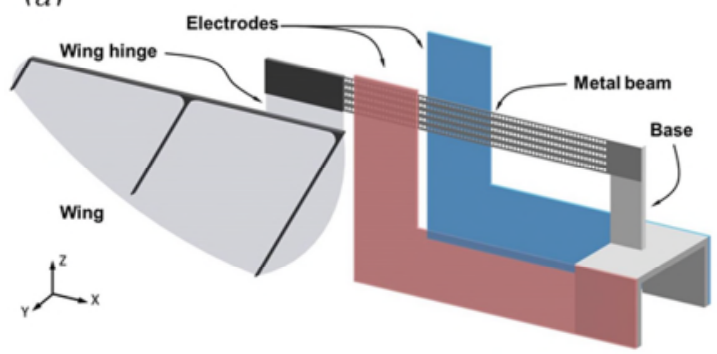

(b)

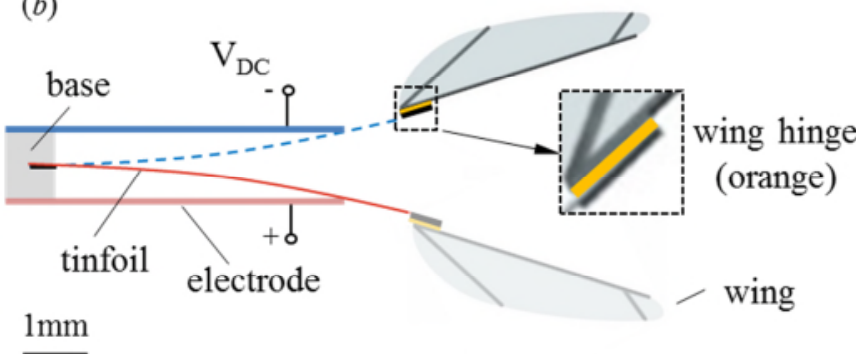

Fig. 1 (a) A conceptual drawing of the actuator. (b) A schematic top view of the actuator showing how the wing flaps and rotates due to electrostatic actuation.

The working principle of the actuator is shown in Fig. 1(b) [1]. In working state, a DC voltage (VDC) is applied to the electrodes, thus the electrostatic field is generated instantaneously. As the $\mathrm{V}_{\mathrm{DC}}$ rising gradually, the wing-beam structure is attracted to one of the electrodes for the reason of electrostatic force unbalance. Once the $\mathrm{V}_{\mathrm{DC}}$ reaches or exceeds the pull-in voltage [4] of the parallel electrodes, the wing-beam structure is excited into the resonance state and strokes at a frequency around its $1^{\text {st }}$ order natural frequency. This kind of vibration pattern is analogous to an insects' wing motion in nature [5]. The actual stroke frequency is determined by the interplay of natural frequency, inertial forces and aerodynamic forces. In this article, considering size and weight limitation of the device, flexure hinge is used to generate passive rotation for the reason of its simple structure and convenience in fabrication. When the metal beam is vibrating, the wing is driven to stroke by the beam via the hinge. Because of the hinge's flexibility, the wing can stroke at an angle of attack. It is noted that the angle of attack is mainly determined by the length, width and material stiffness of the hinge [6], while it may also be influenced by the aerodynamics and inertial loads. Details of the wing hinge will be given in later sections.

\section{Fabrication Process}

The five components mentioned in preceding sections can be divided into three categories: A. frame, consisting of electrodes and base; B. airfoil, including wing and wing hinge, and C. metal beam. Further introduction to the three parts' fabrication process will be presented later and graphical description of that is shown in Fig. 2.

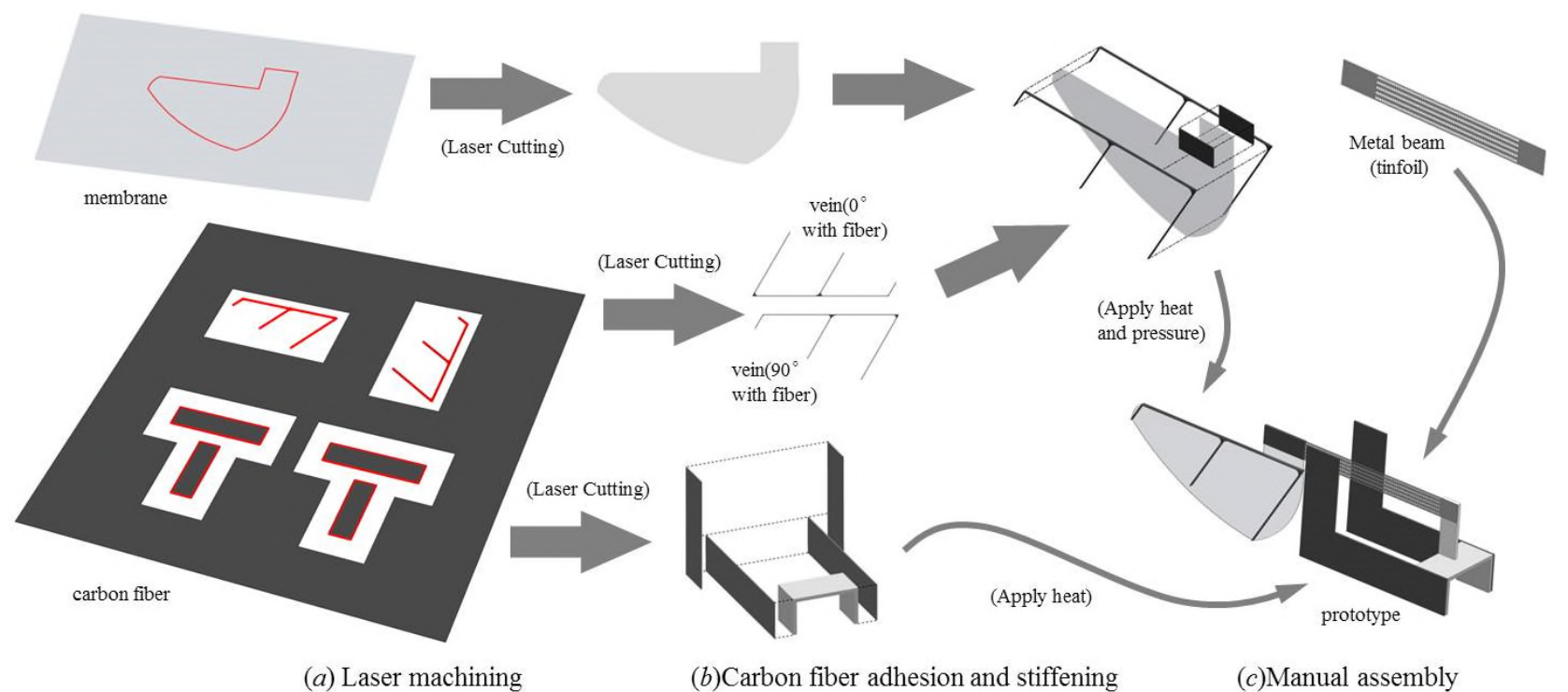

Fig. 2 Creation and assembling process of the actuator, consists of three main steps, (a) laser machining, (b) carbon fiber prepregs stiffening and adhesion, and (c) manual assembly. 
Frame. The frame fabrication process starts with the material called carbon-fiber-reinforced polymer (CFRP), which is also known as prepreg and composed of innumerable unidirectional and parallel carbon fiber filaments along with a plastic resin used as preimpregnation base. After being molded, heated and air-cured, CFRP is stiffened and any structure with desired geometries and high strength-to-weight ratio can be formed in this manner. Stiffened CFRP is extremely rigid in the direction parallel with the filaments $\left(0^{\circ}\right.$ direction), whereas brittle in the direction perpendicular to the filaments $\left(90^{\circ}\right.$ direction). As a consequence, in practical fabrication process, both $0^{\circ}$ direction and $90^{\circ}$ direction small sheets should be cut off from CFRP materials by using laser-machining methodology. Afterwards, two small sheets should be adhered into L-shape electrode by residual resin on surface; two L-shape electrodes are adhered to the base in the same manner. Finally, high temperature is applied to the whole structure to stiffen the prepregs and a frame is formed as a result.

Airfoil. In previous research, wings extracted from drone honey bees were used as airfoils for lightness and convenience in fabrication [1]. However, these wings were slightly curvy on membrane and main vein, which made the wings stroke asymmetrically. Therefore, bees' wings turned out to be inappropriate as airfoils and artificial wings are taken into consideration. Artificial wings are manufactured with the following process: first, an outline including wing membrane and hinge is cut off from a membranous material named BoPET (biaxially-oriented polyethylene terephthalate), whose thickness is $2 \mu \mathrm{m}$, an appropriate size for both wing and hinge. Second, a vein used for geometry retaining purpose is cut off from laminated CFRP, which is pre-machined by laminating two layers of CFRP materials with an angle of $60^{\circ}$. This angle is the angle between the main vein and the branch veins. By doing this, the vein's rigidness is enhanced not only in main vein direction, but also in branch vein direction. The vein is then adhered to the wing membrane by residual resin. Meanwhile, a small piece of CFRP is also adhered to the top of the hinge, which is used to control the hinge length. After heat-pressure applying and stiffening steps, the airfoil fabrication process is finished.

Metal Beam. Compared to our previous study, a thin sheet of tinfoil with numerous holes is processed as the vibration component, instead of parallel super-elastic alloy wires [1]. This modification is made under following considerations. First, since metal beam is a key component on the lift load path, thin sheet could have a better performance in Z-direction force transmission than cylindrical wire. The second is that sheet is more convenient to fabricate than wires by using laser cutting machine. However, sheet may not have an ideal aerodynamic performance as compared with wires, thus a series of throughout holes are punched by laser on the metal beam. With holes array, the beam material acts more like gauze, which has benefit for vibration, increases vibration frequency, and lower pull-in voltage, thus the wing could have a better lift force performance. Table 1 shows the different vibration performance of metal beams with holes and metals beams without holes.

Table 1. Vibration performance of metal beams with holes and metal beams without holes.

\begin{tabular}{c|c|c|c}
\hline metal beams & length $(\mathrm{mm})$ & frequency $(\mathrm{Hz})$ & pull-in voltage $(\mathrm{kV})$ \\
\hline with holes & 6 & 333 & 1.5 \\
without holes & 6 & 273 & 2.1 \\
\hline
\end{tabular}

Assembly. After three crucial components mentioned above are created, they can be integrated into a complete actuator, as shown in 오류! 참조 원본을 찾을 수 없습니다., whose characteristic length is $10 \mathrm{~mm}$ and weight is only $2 \mathrm{mg}$. With high efficiency of laser machining tech, the whole fabrication and assembly process can be finished within one day. The final mass properties of each component and the whole actuator are shown in 오류! 참조 원본을 찾을 수 없습니다.. 
Table 2. Final mass properties for each component.

\begin{tabular}{c|c}
\hline component & mass $(\mathrm{mg})$ \\
\hline frame & 2.7 \\
airfoil & 0.2 \\
metal beam & 0.052 \\
\hline total actuator & $\approx 3$ \\
\hline
\end{tabular}

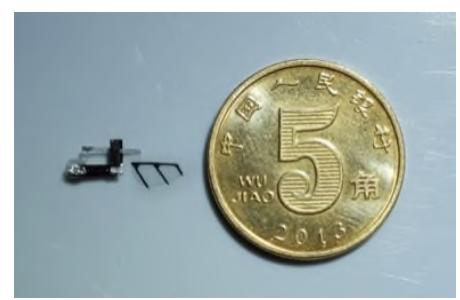

Fig. 3 Photo of the actuator and a coin.

\section{Experiments and Results}

As stroke frequency, stroke amplitude and rotational angle of the wing are extremely crucial for the generation of lift force [7], a benchtop experiment is designed to optimize these characteristics by modifying several key parameters of the actuator, such as electrodes spacing, metal beam length and hinge length. After an actuator with optimal parameters is integrated, a lift force measurement is conducted to demonstrate the actuator's availability for millimeter-scale FMAV.

Benchtop Experiment. Fig. 4(a) shows the setup of the benchtop experiment. Two electrodes are fixed on left and right micro-displacement platform, respectively, which is used to modify the electrodes spacing and electrodes distance from bottom. The wing-beam vibrator is installed between two electrodes. The whole structure is generally similar to the actuator, except that the electrodes spacing and electrodes position is adjustable. Moreover, a high-speed camera is set to capture wing motion from a vertical perspective and to monitor wing kinematic characteristics like amplitude and frequency. After iteratively adjusting the actuator parameters according to the change of kinematic characteristics, some final parameters are determined. With a metal beam length of $6 \mathrm{~mm}$, electrodes spacing of $2 \mathrm{~mm}$, and electrodes distance from bottom of $3 \mathrm{~mm}$, the wing strokes with a swept angle of $80^{\circ}$, a frequency of $80 \mathrm{~Hz}$, and rotates with a rotating angle of $50^{\circ}$. The vertical view of the wing motion in benchtop experiment is shown in Fig. 4(b).

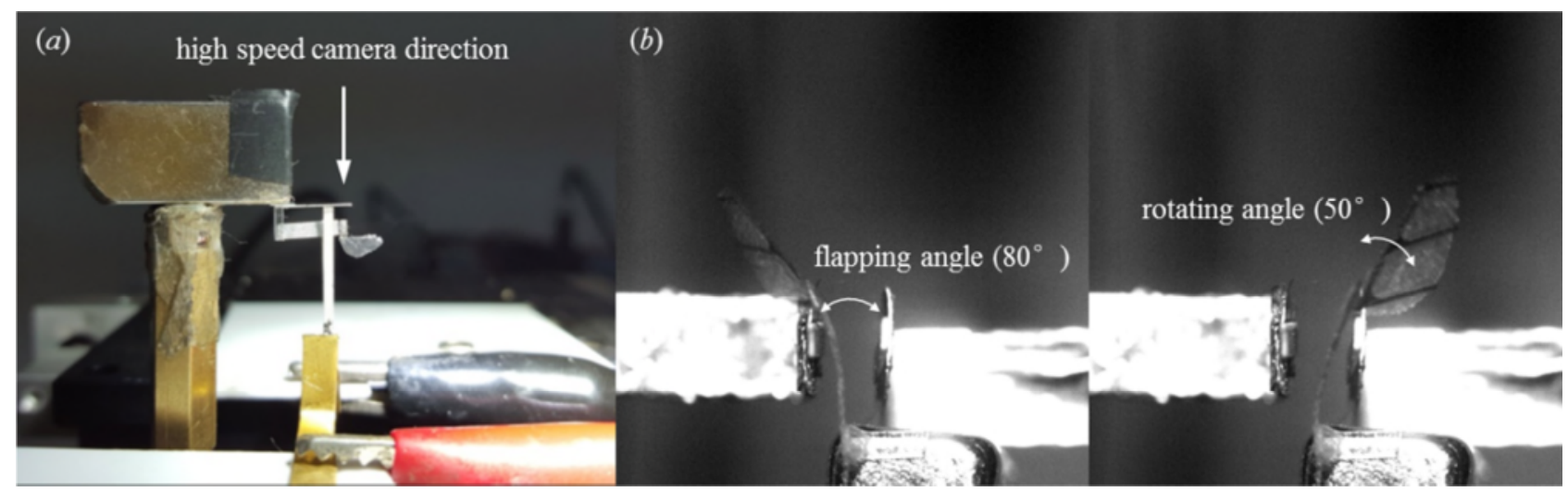

Fig. 4 (a) the setup of the benchtop (b) vertical view of the wing motion.

Lift Force Measurement. A test system mentioned in our previous work [2] is slightly modified to accommodate the size of our actuator. Compared to the previous configuration, a smaller lever and a casing pipe pivot with lubrication aiming at reducing friction are applied on this system.

Fig. 5 gives the instantaneous lift force versus time in two cycles measured by a micro-force sensor, and average lift force. If we take two peaks as one cycle because the wing generates lift force both forward and backward, we can get the flapping frequency about $80 \mathrm{~Hz}$, which matches 
the result of the benchtop experiment well. Moreover, the average lift force generated by the wing is about $1.5 \mathrm{mg}$, or $15 \mu \mathrm{N}$ from Fig. 5, which makes the lift-to-weight ratio reach 0.5 .

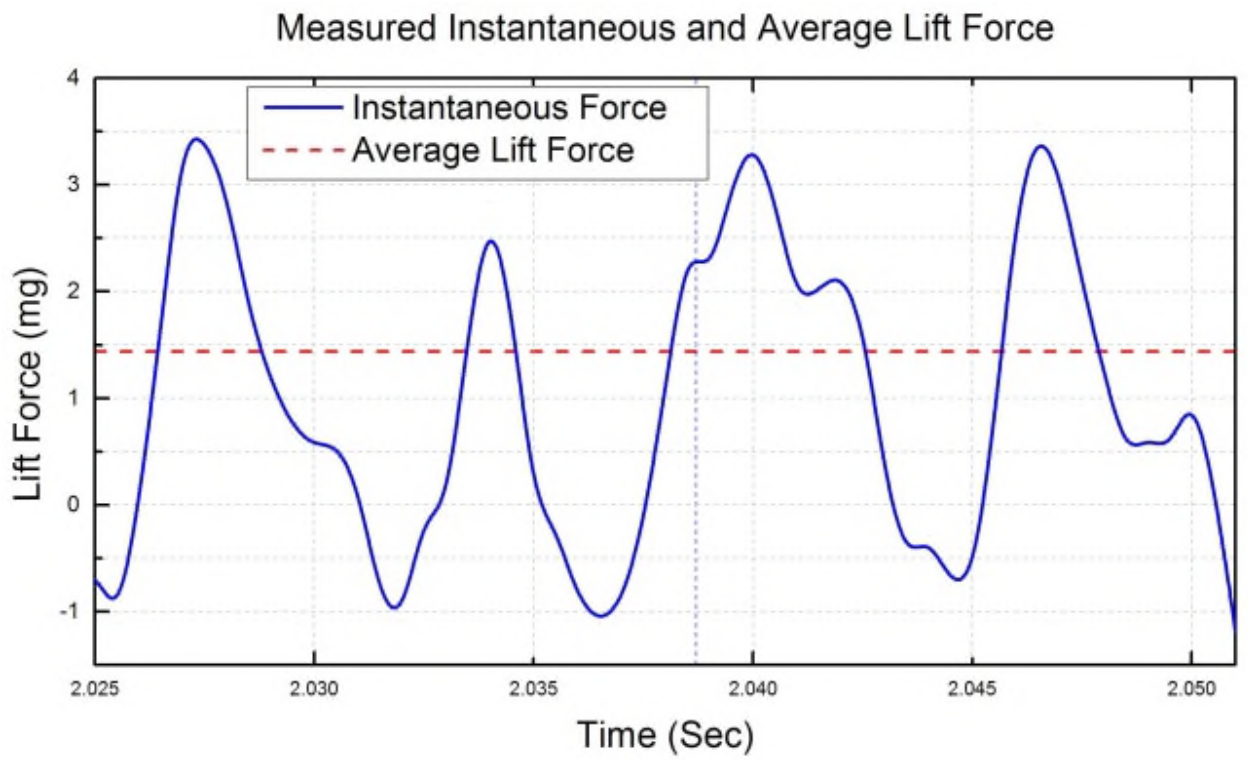

Fig. 5 Instantaneous (blue line) and average lift force (red line) by the actuator as measured using a micro-force sensor.

\section{Conclusion}

This paper presents a millimeter-scale electrostatic flapping-wing actuator with a weight of $3 \mathrm{mg}$, lift force of $15 \mu \mathrm{N}$, and a lift-to-weight ratio of 0.5 . The ratio is two orders of magnitude higher than that of our previous work. Moreover, a new fabrication methodology including laser cutting and the use of carbon-fiber-reinforced polymer material is as also discussed, which can multiply fabrication efficiency and largely lower the mass of prototype. Our future work aims at achieving FMAV's autonomous flight by making further optimization on actuator's structure and force transmission path.

\section{References}

[1] X. Yan, M. Qi, L. Lin. Self-lifting artificial insect wings via electrostatic flapping actuators. in Micro Electro Mechanical Systems (MEMS), 28th IEEE International Conference on. 2015. IEEE.

[2] Z. Liu, et al. Electrostatic flapping wings with pivot-spar brackets for high lift force. in 2016 IEEE 29th International Conference on Micro Electro Mechanical Systems (MEMS). 2016. IEEE.

[3] R. J. Wood, The first takeoff of a biologically inspired at-scale robotic insect. IEEE Trans. Robot. 24(2) (2008) 341-347.

[4] S. Pamidighantam, et al., Pull-in voltage analysis of electrostatically actuated beam structures with fixed-fixed and fixed-free end conditions. J. Micromech. Microeng. 12(4) (2002) 458.

[5] J. A. Miyan, A. W. Ewing, How Diptera move their wings: a re-examination of the wing base articulation and muscle systems concerned with flight. Philosoph. Trans. Royal Soc. B: Biolog. Sci. 311(1150) (1985) 271-302.

[6] R. Malka, et al. Principles of microscale flexure hinge design for enhanced endurance. in Intelligent Robots and Systems (IROS 2014), 2014 IEEE/RSJ International Conference on. 2014. IEEE.

[7] X. Deng, et al., Flapping flight for biomimetic robotic insects: Part I-system modeling. IEEE Trans. Robot. 22(4) (2006) 776-788. 